\section{Magnitude da tuberculose pulmonar na população fronteiriça de Mato Grosso do Sul (Brasil), Paraguai e Bolívia}

\author{
Pulmonary tuberculosis among residents of \\ municipalities in Mato Grosso do Sul State, \\ Brazil, bordering on Paraguay and Bolivia
}

\author{
Magnitud de la tuberculosis pulmonar en la \\ población fronteriza de Mato Grosso do \\ Sul (Brasil), Paraguay y Bolivia
}

1 Universidade Federal de Mato Grosso do Sul, Campo Grande, Brasil.

2 Secretaria de Estado de Saúde de Mato Grosso do Sul, Campo Grande, Brasil. 3 Faculdade de Medicina de Ribeirão Preto, Universidade de São Paulo, Ribeirão Preto, Brasil.

4 Universidade Anhanguera, Campo Grande, Brasil. 5 Universidade Federal do Piauí, Parnaíba, Brasil.

\section{Correspondência} M. Marques

Universidade Federal de Mato Grosso do Sul. Cidade Universitária $s / n$, Campo Grande, MS 79070-900, Brasil. marli.marques2008@ gmail.com

\begin{abstract}
This study analyzed the epidemiological profile of pulmonary tuberculosis from 2007 to 2010 in municipalities (counties) in Mato Grosso do Sul State, Brazil, that border on Paraguay and Bolivia. In the border region, the incidence rate (49.1/100,000 inhabitants), mortality rate (4.0/100,000 inhabitants), and treatment dropout rate (11.3\%) were $1.6,1.8$, and 1.5 times higher than in the non-border region. Among indigenous individuals in the border region, the rates for incidence (253.4/100,000 inhabitants), mortality (11.6/100,000 inhabitants), and HIVITB co-infection (1.9/100,000 inhabitants) were 6.4, 3.2, and 1.9 times higher than in nonindigenous individuals in this region. Living in the border regions was inversely associated with HIVITB co-infection. Indigenous ethnicity was associated statistically with not abandoning $T B$ treatment. The study concludes that the population residing in these municipalities along the border is exposed to high risk of pulmonary TB and TB mortality and treatment dropout, thus requiring special health surveillance interventions.
\end{abstract}

Pulmonary Tuberculosis; South American Indians; Border Areas
Marli Marques 1,2

Antonio Ruffino-Netto ${ }^{3}$

Ana Maria Campos Marques 4

Sonia Maria Oliveira de Andrade 1 Baldomero Antonio Kato da Silva 1,5 Elenir Rose Jardim Cury Pontes 1

\section{Resumo}

Este estudo analisou a magnitude da tuberculose pulmonar no período de 2007 a 2010 em municípios sul-mato-grossenses fronteiriços ao Paraguai e à Bolívia. Na região de fronteira, as taxas de incidência (49,1/100 mil habitantes), de mortalidade (4,0/100 mil) e de abandono do tratamento (11,3\%) foram 1,6, 1,8 e 1,5 vez maiores do que na região não fronteiriça. Entre indígenas da fronteira, as taxas de incidência $(253,4 / 100$ mil habitantes), mortalidade (11,6/100 mil) $e$ coinfecção por HIV (1,9/100 mil) foram, respectivamente, 6,4, 3,2 vezes e 1,9 vez maiores do que entre os não indígenas nesta região. Estar na região de fronteira revelou-se fator de proteção contra coinfecção por HIV. Constatou-se associação entre ser indígena e não abandonar o tratamento. Conclui-se que a população residente nesses municípios de fronteira vivencia elevado risco de adoecimento, de morte e de abandono do tratamento de tuberculose pulmonar, o que requer ações diferenciadas de vigilância em saúde.

Tuberculose Pulmonar; Índios Sul-Americanos; Áreas de Fronteira 


\section{Introdução}

A despeito dos avanços no diagnóstico e tratamento, a tuberculose permanece como um dos principais agravos à saúde em âmbito global 1 e como uma das mais importantes causas de morte nos países em desenvolvimento ${ }^{2}$. Somente em 2012 foram notificados 8,6 milhões de casos novos e 1,3 milhão de mortes, dentre as quais 0,3 milhão associadas ao vírus da imunodeficiência humana (HIV) ${ }^{3}$. No Brasil, a doença segue o padrão mundial de declínio, embora em alguns segmentos populacionais seus níveis de concentração superem a média nacional. Na população negra, a incidência é o dobro da nacional; na população indígena, é quatro vezes maior do que a do país. Entre os portadores de HIV a incidência é 30 vezes maior do que a nacional. Na população que vive em situação de rua, supera em 50 a 60 vezes a taxa registrada na população geral 4 .

As fronteiras têm sido historicamente espaços marginais, periféricos e carentes de integração socioeconômica 5 , porém vêm passando por profundas transformações, com vistas ao desenvolvimento regional. Nesse contexto, a saúde comparece como elemento vital e estratégico, por constituir importante vetor de integração entre sociedades e cidadãos fronteiriços. Nas fronteiras da América do Sul, a dinamização da economia, o combate integrado à violência, a criação de alternativas de inclusão social, as ações de educação e as questões relativas à segurança nacional são considerados essenciais para o sucesso das políticas de saúde 6 .

Localizado na Região Centro-oeste brasileira, Mato Grosso do Sul faz fronteira com a Bolívia e o Paraguai, em uma extensão total de $1.578 \mathrm{~km}$, ao longo da qual estão localizados 12 municípios: Antônio João, Aral Moreira, Caracol, Coronel Sapucaia, Japorã, Mundo Novo, Paranhos, Ponta Porã, Porto Murtinho, Sete Quedas, Corumbá e Bela Vista (Figura 1). Segundo o Censo Demográfico de 2010, Mato Grosso do Sul contava com 2.449 .024 habitantes, $12,5 \%$ dos quais viviam nos municípios fronteiriços e 87,5\% nos demais municípios. Na região de fronteira, a população residente era de 305.953 habitantes, 13.524 dos quais $(4,4 \%)$ se autodeclararam indígenas 7 .

Considerando as particularidades ambientais, sociais, econômicas, demográficas e culturais que contribuem para a diversidade das doenças e de seu comportamento em cada região, este estudo se propôs a descrever a magnitude da tuberculose pulmonar no período de 2007-2010 nos 12 municípios sul-mato-grossenses situados na linha de fronteira com o Paraguai e a Bolívia.

\section{População e métodos}

Procedeu-se a um estudo epidemiológico retrospectivo, descritivo, de dados secundários e de base populacional de todos os casos e óbitos por tuberculose pulmonar notificados de janeiro de 2007 a dezembro de 2010, ocorridos nos $12 \mathrm{mu}$ nicípios sul-mato-grossenses situados na linha de fronteira com o Paraguai e a Bolívia e nos demais 66 municípios do estado. A coleta dos dados considerou sua distribuição por região (fronteiriça e não fronteiriça) e por raça/cor (indígena e não indígena).

Os dados populacionais utilizados foram obtidos nas estimativas para o período de 2007 a 2009 e no Censo Demográfico de 2010, ambos do Instituto Brasileiro de Geografia e Estatística (Sistema IBGE de Recuperação Automática. Censo Demográfico e Contagem Populacional. http:// www.sidra.ibge.gov.br). Para o cálculo da população indígena do estado tomou-se sua contagem expressa nos censos de 2000 e de 2010 e calculouse sua taxa média de crescimento geométrico estadual para o período 2001-2009. Essa taxa média foi então aplicada a cada município para estimar as suas populações indígenas.

Os dados referentes a casos de tuberculose pulmonar notificados no período de 2007-2010 foram obtidos no Sistema de Informação de Agravos de Notificação (SINAN) da Secretaria de Estado de Saúde de Mato Grosso do Sul. Foram considerados casos novos as notificações em que a variável "tipo de entrada" estivesse preenchida com a categoria "caso novo" ou "não sabe" e a forma clínica preenchida com a categoria "pulmonar e pulmonar + extrapulmonar". Nesse universo foi avaliada a presença de sorologia "positiva” para HIV e situação de encerramento como "abandono" 7. Foram incluídos todos os casos novos pulmonares, independentemente de coinfecção por HIV ou de abandono de tratamento, correspondentes ao período de janeiro de 2007 a dezembro de 2010. Foram excluídos os registros duplicados e aqueles que tiveram "mudança de diagnóstico" como situação de encerramento.

Os registros de óbitos por tuberculose pulmonar foram obtidos no Sistema de Informação de Mortalidade (SIM) da Secretaria de Estado de Saúde de Mato Grosso do Sul, considerando-se aqueles registrados no período de 2007-2010 que identificaram como causa a tuberculose respiratória, de classificação CID-10(005) (Classificação Internacional de Doenças - 10a revisão).

Os casos novos, os de coinfecção por HIV, os de abandono do tratamento e os óbitos foram analisados de forma agrupada quanto à classificação em indígenas e não indígenas e segundo a região de residência (fronteira e não fronteira). 


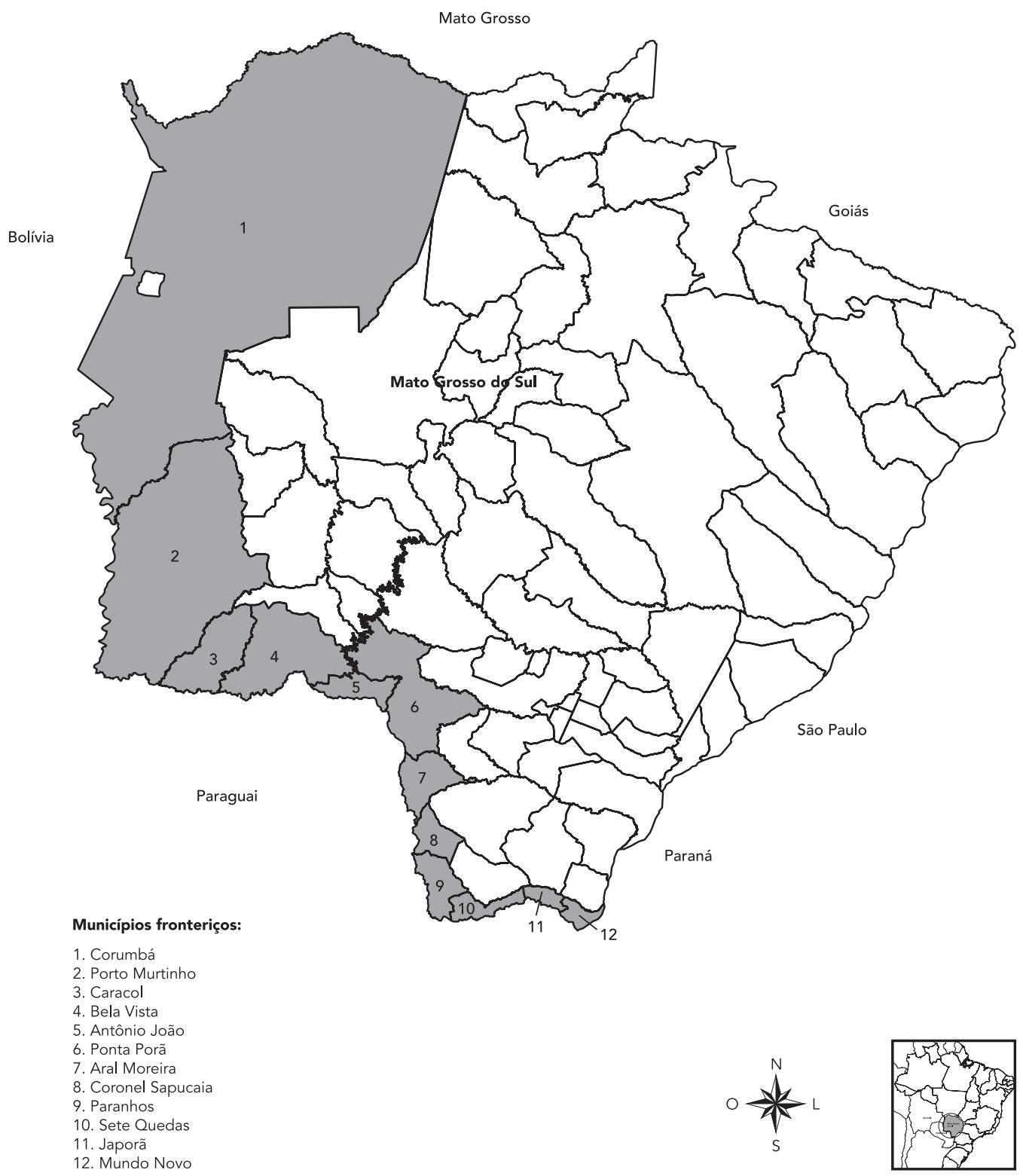

No grupo não indígena foram incluídas as demais categorias de raça/cor, a saber: branca, negra, amarela, parda e ignorada. No grupo de fronteira foram incluídos os casos residentes nos 12 municípios fronteiriços ao Paraguai ou à Bolívia.

Para o cálculo das taxas de incidência, mortalidade específica e coinfecção por HIV (por 100 mil habitantes), multiplicou-se o número de casos por 100 mil e dividiu-se o resultado pelo total de pessoas/ano de exposição, referente a cada variável analisada e ao período de estudo (2007-2010). Para o abandono de tratamento, expresso em porcentagem, utilizou-se o número de casos multiplicado por 100, sendo o resultado dividido pelo total de casos novos de tuberculose pulmonar notificados em cada grupo ou região 
analisados. Adotou-se um intervalo de 95\% de confiança (IC95\%) para todas as taxas. Para a comparação das taxas entre as regiões (fronteira e não fronteira) e grupos populacionais (indígena e não indígena), utilizou-se o teste qui-quadrado para comparação de proporções e cálculo de risco relativo com o respectivo IC95\%, seguido de análise estratificada de Mantel-Haenszel com cálculo de risco relativo ajustado e IC95\%, a fim de verificar confundimento ou interação entre estas variáveis. Adotou-se um nível de $5 \%$ de significância ( $\mathrm{p} \leq 0,05)$.

O estudo foi aprovado pelo Comitê de Ética em Pesquisa da Universidade Federal de Mato Grosso do Sul e pela Comissão Nacional de Ética em Pesquisa do Conselho Nacional de Saúde (CONEP/CNS) (pareceres 141.949/2012 e 507.049/2013, respectivamente).

\section{Resultados}

De 2007-2010 foram notificados 2.981 casos novos e 218 óbitos por tuberculose pulmonar em Mato Grosso do Sul. A investigação sorológica de HIV foi realizada em 1.951 casos $(65,4 \%)$, com resultado positivo em 253 (8,5\%). O abandono de tratamento foi avaliado em todos os casos novos, incluindo aqueles com desfecho ignorado, perfazendo $4 \%$ (124/2.981).

Embora nos 12 municípios fronteiriços pesquisados vivam apenas $12,5 \%$ da população sulmato-grossense, neles são registrados aproximadamente $20 \%$ dos casos novos de tuberculose pulmonar, mais de $25 \%$ dos óbitos por esta causa e abandonos de tratamento, e pouco mais de $4,7 \%$ de coinfecção por HIV. Os números absolutos de casos e as taxas por localidade e grupo populacional estão na Tabela 1 . Na região de fronteira, as taxas de incidência (49,1/100 mil hab.), de mortalidade (4,0/100 mil) e de abandono do tratamento (11,3\%) foram, respectivamente, 1,6, 1,8 e 1,5 vez maior do que na região não fronteiriça.

Na população indígena da região de fronteira, as taxas de incidência, mortalidade e coinfecção por HIV foram, respectivamente, 6,4, 3,2 e 1,9 vezes maiores do que na população não indígena desta região, ao passso que a taxa de abandono foi 2,5 vezes menor.

Os intervalos de confiança para as taxas de incidência, mortalidade específica e coinfecção por HIV e para os percentuais de abandono em Mato Grosso do Sul, por região e grupo populacional, estão apresentados na Tabela 2.

Tabela 1

Distribuição de casos, óbitos, coinfecção por HIV e abandono do tratamento da tuberculose pulmonar e taxas médias de incidência, mortalidade específica e de coinfecção por HIV (por 100 mil habitantes) e percentuais de abandono de tratamento, por região e grupo populacional. Mato Grosso do Sul, Brasil, 2007-2010.

\begin{tabular}{|c|c|c|c|c|c|c|}
\hline \multirow[t]{2}{*}{ Variáveis } & \multicolumn{2}{|c|}{ Fronteira } & \multicolumn{2}{|c|}{ Não fronteira } & \multicolumn{2}{|c|}{ Mato Grosso do Sul } \\
\hline & $\mathbf{n}$ & Taxa & $\mathbf{n}$ & Taxa & n & Taxa \\
\hline \multicolumn{7}{|c|}{ Taxa média de incidência de tuberculose pulmonar } \\
\hline Indígenas & 131 & 253,4 & 387 & 169,4 & 518 & 184,9 \\
\hline Não indígenas & 451 & 39,7 & 2012 & 25,0 & 2463 & 26,8 \\
\hline População total & 582 & 49,1 & 2399 & 28,9 & 2981 & 31,5 \\
\hline \multicolumn{7}{|c|}{ Taxa média de mortalidade específica por tuberculose pulmonar } \\
\hline Indígenas & 6 & 11,6 & 25 & 10,9 & 31 & 11,1 \\
\hline Não indígenas & 41 & 3,6 & 146 & 1,8 & 187 & 2,0 \\
\hline População total & 47 & 4,0 & 171 & 2,1 & 218 & 2,3 \\
\hline \multicolumn{7}{|c|}{ Taxa média de coinfecção por HIV } \\
\hline Indígenas & 1 & 1,9 & 6 & 2,6 & 7 & 2,5 \\
\hline Não indígenas & 11 & 1,0 & 235 & 2,9 & 246 & 2,7 \\
\hline População total & 12 & 1,0 & 241 & 2,9 & 253 & 2,7 \\
\hline \multicolumn{7}{|c|}{ Abandono do tratamento de tuberculose pulmonar (\%) } \\
\hline Indígenas & 7 & 5,3 & 14 & 3,6 & 21 & 4,1 \\
\hline Não indígenas & 59 & 13,1 & 180 & 8,9 & 239 & 9,7 \\
\hline Total de abandonos & 66 & 11,3 & 194 & 8,1 & 260 & 8,7 \\
\hline
\end{tabular}

Nota: dados provenientes do Sistema de Informação de Agravos de Notificação (SINAN), Secretaria de Estado de Saúde de Mato Grosso do Sul, 2007-2010; Instituto Brasileiro de Geografia e Estatística (IBGE) (Censos Demográficos 2000 e 2010). 
Taxas médias de incidência, mortalidade específica e coinfecção por HIV (por 100 mil habitantes), percentuais de abandono do tratamento de tuberculose pulmonar e intervalos de 95\% de confiança (IC95\%), por região e grupo populacional. Mato Grosso do Sul, Brasil, $2007-2010$.

\begin{tabular}{|c|c|c|c|c|c|c|}
\hline \multirow[t]{2}{*}{ Variáveis } & \multicolumn{2}{|c|}{ Fronteira } & \multicolumn{2}{|c|}{ Não Fronteira } & \multicolumn{2}{|c|}{ Mato Grosso do Sul } \\
\hline & Taxa & $\mathrm{IC} 95 \%$ & Taxa & IC95\% & Taxa & IC95\% \\
\hline \multicolumn{7}{|c|}{ Taxa média de incidência de tuberculose pulmonar } \\
\hline Indígenas & 253,4 & $251,2-255,6$ & 169,4 & $168,7-170,1$ & 184,9 & $184,2-185,6$ \\
\hline Não indígenas & 39,7 & $39,6-39,8$ & 25,0 & $24,9-25,1$ & 26,8 & $26,7-26,9$ \\
\hline População total & 49,1 & $49,0-49,2$ & 28,9 & $28,8-29,0$ & 31,5 & $31,0-31,6$ \\
\hline \multicolumn{7}{|c|}{ Taxa média de mortalidade específica por tuberculose pulmonar } \\
\hline Indígenas & 11,6 & $11,5-11,7$ & 10,9 & $10,8-11,0$ & 11,1 & $11,0-11,2$ \\
\hline Não indígenas & 3,6 & $3,5-3,7$ & 1,8 & $1,7-1,9$ & 2,0 & $1,9-2,1$ \\
\hline População total & 4,0 & $3,9-4,1$ & 2,1 & $2,0-2,2$ & 2,3 & $2,2-2,4$ \\
\hline \multicolumn{7}{|c|}{ Taxa média de coinfecção por HIV } \\
\hline Indígenas & 1,9 & $1,8-2,0$ & 2,6 & $2,5-2,7$ & 2,5 & $2,4-2,6$ \\
\hline Não indígenas & 1,0 & $0,9-1,1$ & 2,9 & $2,8-3,0$ & 2,7 & $2,6-2,8$ \\
\hline População total & 1,0 & $0,9-1,1$ & 2,9 & $2,8-3,0$ & 2,7 & $2,6-2,8$ \\
\hline \multicolumn{7}{|c|}{ Abandono do tratamento de tuberculose pulmonar (\%) } \\
\hline Indígenas & 5,3 & $4,5-6,2$ & 3,6 & $3,3-3,9$ & 4,1 & $3,8-4,4$ \\
\hline Não indígenas & 13,1 & $11,9-14,2$ & 8,9 & $8,6-9,3$ & 9,7 & $9,3-10,1$ \\
\hline Total de abandonos & 11,3 & $10,5-12,2$ & 8,1 & $7,8-8,4$ & 8,7 & $8,4-9,0$ \\
\hline
\end{tabular}

Nota: dados provenientes do Sistema de Informação de Agravos de Notificação (SINAN), Secretaria de Estado de Saúde de Mato Grosso do Sul, 2007-2010; Instituto Brasileiro de Geografia e Estatística (IBGE) (Censos Demográficos 2000 e 2010).

Os valores de p, na comparação das taxas entre regiões (fronteira e não fronteira) e grupos populacionais (indígena e não indígena), estão na Tabela 3.

Na população indígena do estado, as taxas de incidência (184,9/100 mil habitantes) e de mortalidade (11,1/100 mil habitantes) foram, respectivamente, 6,7 e 5,2 vezes maiores do que na população não indígena. A taxa de abandono $(9,7 \%)$ entre os não indígenas foi maior do que entre os indígenas, com diferença estatisticamente significativa $(p<0,001)$.

Estar na região de fronteira foi fator de proteção contra coinfecção por HIV.

Constatou-se associação entre ser indígena e não abandonar o tratamento de tuberculose pulmonar, ao passo que não foi verificada associação entre ser ou não indígena e coinfeção por HIV.

\section{Discussão}

O conhecimento da relação entre eventos de saúde e doença e o espaço geográfico é essencial para o adequado diagnóstico da situação de saúde, permitindo o desenvolvimento de ações eficazes para o controle de doenças. Os diferentes luga- res se configuram como resultados de diferentes acúmulos de situações históricas, ambientais e sociais, que promovem condições particulares para a produção de doenças 8 . No caso da tuberculose, focalizar a sua distribuição em duas regiões do estado (fronteiriça e não fronteiriça) permitiu constatar a existência de uma área crítica, evidenciando elementos que podem contribuir para a formulação de propostas de intervenção mais efetivas, a exemplo dos achados de um estudo sobre distribuição espacial realizado em Ribeirão Preto, São Paulo ${ }^{9}$.

Em Mato Grosso do Sul, evidenciou-se uma taxa de incidência de tuberculose pulmonar de 31,5/100 mil habitantes. Considerando-se que em 2010 a taxa nacional para todas as formas de tuberculose foi de 37,7/100 mil e que a tuberculose pulmonar foi responsável por cerca de 85,9\% deste total 10 , estima-se que a taxa de incidência média no território nacional seja de 32,4/100 mil habitantes, superior portanto à de Mato Grosso do Sul. Similarmente, verificou-se que a taxa estadual foi menor do que as encontradas em outras 12 unidades federadas, figurando o Estado do Rio de Janeiro em primeiro lugar, com 60,7/100 mil habitantes ${ }^{10}$. Mesmo na Região Centro-oeste, as taxas de incidência também estão distantes de forma significativa entre as quatro unidades da 
Tabela 3

Riscos relativos brutos e ajustados das taxas médias de incidência e mortalidade específica, coinfecção por HIV (por 100 mil habitantes) e percentuais de abandono do tratamento, por região e grupo populacional. Mato Grosso do Sul, Brasil, 2007-2010.

\begin{tabular}{|c|c|c|c|c|}
\hline Variáveis & Taxas & Valor de $\mathrm{p}$ & RR bruto (IC95\%) & RR ajustado (IC95\%) \\
\hline \multicolumn{5}{|c|}{ Taxa média de incidência de tuberculose pulmonar } \\
\hline Fronteira & 49,1 & $<0,001$ & $1,70(1,55-1,86)$ & $1,57(1,43-1,72)$ * \\
\hline Não fronteira & 29,0 & & & \\
\hline Indígenas & 184,9 & $<0,001$ & $6,90(6,28-7,59)$ & $6,68(6,08-7,35) * *$ \\
\hline Não indígenas & 26,8 & & & \\
\hline \multicolumn{5}{|c|}{ Taxa média de mortalidade específica } \\
\hline Fronteira & 4,0 & $<0,001$ & $1,92(1,39-2,65)$ & $1,80(1,31-2,49)$ * \\
\hline Não fronteira & 2,1 & & & \\
\hline Indígenas & 11,1 & $<0,001$ & $5,44(3,72-7,96)$ & $5,17(3,54-7,56) * \star$ \\
\hline Não indígenas & 2,0 & & & \\
\hline \multicolumn{5}{|c|}{ Taxa média de coinfecção por HIV } \\
\hline Fronteira & 1,0 & $<0,001$ & $0,35(0,19-0,62)$ & $0,35(0,19-0,62)$ * \\
\hline Não fronteira & 2,9 & & & \\
\hline Indígenas & 2,5 & 0,859 & $0,93(0,44-1,98)$ & $0,98(0,46-2,07) * \star$ \\
\hline Não indígenas & 2,7 & & & \\
\hline \multicolumn{5}{|c|}{ Abandono do tratamento de tuberculose pulmonar (\%) } \\
\hline Fronteira & 11,3 & 0,013 & $1,40(1,08-1,83)$ & $1,46(1,12-1,90)$ * \\
\hline Não fronteira & 8,1 & & & \\
\hline Indígenas & 4,1 & $<0,001$ & $0,42(0,27-0,65)$ & $0,41(0,26-0,63)$ \\
\hline Não indígenas & 9,7 & & & \\
\hline
\end{tabular}

IC95\%: intervalo de 95\% de confiança; RR: risco relativo.

Nota: teste qui-quadrado seguido de análise estratificada de Mantel-Haenszel. Não houve confundimento ou interação entre as variáveis "grupo

populacional" e "região".

* Estratificado por grupo populacional (indígena e não indígena);

** Estratificado por região (fronteira e não fronteira).

federação que a compõe, tendo o Distrito Federal a menor delas (9,7/100 mil) 10. Essa distribuição heterogênea foi também observada em Mato Grosso do Sul: na região fronteiriça, a incidência, de 49,1/100 mil habitantes, foi 1,6 vez superior à verificada na região não fronteiriça.

A faixa de fronteira brasileira configura-se como pouco desenvolvida, historicamente abandonada pelo Estado e marcada por dificuldades de acesso a bens e serviços públicos, por falta de coesão social e por inobservância de condições de cidadania ${ }^{6}$. Na linha da fronteira internacional de Mato Grosso do Sul, esse retardo decorre do modelo tradicional e anacrônico de ocupação extensiva, primitiva e predatória de seus recursos naturais 11 . Peculiaridades territoriais e históricas que remontam ao século XVI fizeram com que a região se mantivesse à margem do controle estatal até o século XIX, dificultado tanto pela extensão territorial quanto pela baixa densidade populacional, além da existência de con- flitos com populações indígenas. A despeito de sua inclusão nas políticas públicas em tempos recentes, apresenta ainda defasagem nos indicadores de desenvolvimento humano, usualmente baixos 12 .

Os municípios fronteiriços de Mato Grosso do Sul, segundo Peiter 13, caracterizam-se pela combinação de elevada incidência de tuberculose e AIDS, e carência de atendimento de saúde em ambos os lados da fronteira, com fluxos transfronteiriços constantes e/ou presença de populações indígenas vulneráveis 13 .

Na Bolívia, a taxa de tuberculose pulmonar foi de 59,9/100 mil habitantes em 2010 14, evidenciando o elevado risco de adoecimento da população que transita, em grande fluxo, entre esse país e o Município de Corumbá, seja com destino a outras localidades ou para desenvolver atividades ocupacionais diárias, aumentando o risco de propagação. Outro estudo revelou que os bolivianos que passaram a residir e trabalhar 
no estado têm alta taxa de adoecimento por tuberculose, o que contribui para manter elevada a taxa nos locais em que passaram a residir 15.

No Paraguai, a taxa de incidência de todas as formas de tuberculose foi de 32,8/100 mil habitantes em 2010; a de incidência de infecção por HIV, por sua vez, foi de 15,1/100 mil em 200914 A influência da situação de fronteira de Mato Grosso do Sul com o Paraguai se destaca nas cidades gêmeas de Ponta Porã/Pedro Juan Caballero e Coronel Sapucaia/Capitán Bado, não só em decorrência do livre acesso binacional, das atividades econômicas comuns e do turismo de compras em Pedro Juan Caballero, mas também por razões de moradia, trabalho e busca de serviços e de assistência, tanto por brasileiros quanto por paraguaios e cidadãos com dupla cidadania 16 . Esse livre trânsito entre as cidades gêmeas não impede que barreiras de acesso aos serviços de saúde dificultem o diagnóstico da tuberculose. Na fronteira Brasil/Paraguai/Argentina, SilvaSobrinho et al. 17 identificaram vários fatores dificultantes, incluindo diferenças nos sistemas de saúde (com estruturas, processos e resultados diferentes) e aspectos antropológicos específicos das populações envolvidas.

Estudo utilizando dados do suplemento saúde da Pesquisa Nacional por Amostra de Domicílios (PNAD), realizada em 2008, sobre autorrelatos de tuberculose e determinantes sociais para regiões metropolitanas do Brasil, constatou associação entre renda inferior a meio salário mínimo por membro da família e ocorrência de tuberculose 18. Os autores concluíram que a privação e as barreiras de acesso a recursos básicos constituíram os fatores mais importantes para a ocorrência da doença.

Um estudo comparativo entre condições de vida e distribuição da carga da tuberculose em bairros de Ribeirão Preto, evidenciou que as áreas com maior número de casos coincidiram com as de condições de vida baixa e intermediária, sugerindo a existência de bolsões de pobreza distribuídos nos mais diversos bairros do município 19.

Outro aspecto relevante diz respeito às características étnicas, visto que Mato Grosso do Sul abriga a segunda maior população indígena do país, presente na maioria dos municípios e perfazendo $4,3 \%$ da população da linha de fronteira e $2,8 \%$ nos demais municípios. Verifica-se taxa de incidência 6,7 vezes maior do que entre os não indígenas, havendo associação significativa entre ser indígena e ter tuberculose pulmonar, corroborando os achados de Cunha et al. ${ }^{20} \mathrm{com}$ base na genotipagem de Mycobacterium tuberculosis. Esses autores constataram elevada taxa de transmissão recente nessa população e vín- culo epidemiológico estabelecido entre $30 \%$ dos casos, confirmando a propagação interpessoal da doença. Inúmeros trabalhos apontam maior vulnerabilidade dos povos indígenas ao adoecimento por tuberculose em comparação com outros segmentos da população $21,22,23,24,25$, o que se agrava frente às condições de pobreza, falta ou limitação de acesso aos serviços de saúde, uso abusivo de álcool, deficiências nutricionais e presença de comorbidades 26,27 .

Os achados da nossa pesquisa são coerentes com uma revisão sobre a tuberculose em indígenas empreendida globalmente. Tal estudo revelou que a carga da tuberculose é elevada e desproporcional, com grande variação entre grupos e regiões. Os grupos indígenas com maior carga da doença estão localizados em pequenas regiões da América Latina 28.

Embora a taxa de incidência de tuberculose pulmonar em Mato Grosso do Sul seja próxima da nacional 11, indicando risco de adoecimento semelhante na população brasileira e na sul-mato-grossense, a avaliação de universos menores e populações distintas evidenciou a região de fronteira do estado e a população indígena como sendo a de maior risco de adoecimento e morte por tuberculose pulmonar.

Melhorias nas condições sanitárias e das ações do Programa de Controle da Tuberculose reduziram o risco de infecção em muitos países. No entanto, o retardo no diagnóstico aumenta a gravidade da doença e potencializa a disseminação da infecção na comunidade, elevando a mortalidade 29,30.

Segundo a Organização Mundial da Saúde (OMS) 31, em 2010 a taxa de mortalidade por "todas as formas" de tuberculose foi de 2,6/100 mil habitantes no Brasil, 4,2/100 mil no Paraguai e 20/100 mil na Bolívia, em contraste com 2,2/100 mil no continente americano. Em vista da proximidade geográfica com o Paraguai e a Bolívia, os municípios situados na linha de fronteira focalizados em nosso trabalho apresentaram taxa de mortalidade de 4,0/100 mil habitantes que, embora restringindo-se às formas pulmonares, aproximaram-se dos verificados no Paraguai.

A mortalidade por tuberculose pulmonar em Mato Grosso do Sul (2,3/100 mil habitantes) foi portanto menor do que a registrada no Brasil para todas as formas da doença no período de 2001 a 2010, embora superando as taxas de oito estados brasileiros neste último ano 10 .

As discrepâncias observadas entre as taxas da região de fronteira e da área de não fronteira e entre a população indígena e a não indígena foram estatisticamente significativas ( $p<0,001)$, indicando que a população sul-mato-grossense está sujeita a elevado risco de morte por tuberculose, 
1,8 vez maior na zona fronteiriça do que nos demais municípios e 5,2 vezes maior em indígenas do que em não indígenas. Esses resultados são compatíveis com os obtidos para a população indígena do Município de São Gabriel da Cachoeira, na linha de fronteira com a Colômbia e a Venezuela, onde a mortalidade por todas as formas de tuberculose alcançou 13,24/100 mil habitantes no período de 1997 a 2007 32. Elevadas taxas de mortalidade na população indígena podem ser atribuídas às condições de vida, principalmente quando é alta a densidade intradomiciliar, além das condições nutricionais e sociossanitárias precárias e das deficiências nas ações de controle 33,34,35,36. Em muitas localidades de fronteira, apesar da contiguidade territorial, operam sistemas de saúde diferentes 37,38 . A implementação de estratégias de saúde que visem a integrá-los é viável no que diz respeito a ações básicas de saúde 38 , o que contribuiria para reduzir a mortalidade decorrente das dificuldades de acesso aos serviços de atenção básica.

Os municípios situados na linha da fronteira internacional de Mato Grosso do Sul merecem receber intervenções de programas governamentais que não se limitem às ações de controle da doença. Requerem-se programas que resultem em redução das desigualdades sociais, permitindo alcançar a diminuição almejada nos indicadores da doença, quebrando o ciclo que alimenta a pobreza e a exclusão social, tendo-se em vista o impacto negativo exercido pela tuberculose no crescimento econômico e no desenvolvimento social do estado e da Região Centro-oeste 39.

A infecção por HIV não só tem contribuído para um crescente número de casos de tuberculose como também tem sido um dos principais fatores para a elevação da mortalidade entre pacientes coinfectados 31 .

A testagem do HIV em Mato Grosso do Sul alcançou, no período investigado, $60 \%$, variando entre os municípios de fronteira de $30 \%$ a $100 \%$, com média inferior a 50\%. Em dois municípios que responderam por $58 \%$ dos casos de tuberculose pulmonar da região, a investigação do HIV foi inferior a $40 \%$. Tanto na linha de fronteira como na região não fronteiriça, a coinfecção por HIV esteve presente em 2,5/100 mil habitantes indígenas, índice este obtido em investigação de mais de $50 \%$ dos casos de tuberculose pulmonar registrados entre indígenas. Tal achado é preocupante, e provavelmente se verifique também em outras comunidades indígenas do país, permanecendo subdetectado em vista do baixo índice de investigação (10\%) 25,32.

Apesar dessas variações, a taxa estadual de coinfecção por HIV foi de 2,7/100 mil habitantes, embora menor na região de fronteira $(1,0 / 100$ mil). Em 2010, as taxas para todas as formas de tuberculose foram de 3,2 e 3,7/100 mil em Mato Grosso do Sul e no Brasil, respectivamente 4 . Segundo dados da OMS, em 2010 essa taxa foi inferior a 1/100 mil no Paraguai, de 4,5/100 mil na Bolívia e de 3,7 / 100 mil nas Américas 31. Na linha de fronteira pesquisada no presente trabalho, essa taxa é provavelmente menor, considerando-se que os dados utilizados se restringiram aos casos de tuberculose pulmonar e que no Brasil as formas extrapulmonares representaram aproximadamente $14 \%$ dos casos novos 10 .

Em nosso estudo, a maior mortalidade verificada entre residentes na linha de fronteira parece não decorrer da coinfecção com HIV, corroborando análises estatísticas que indicam que morar na região de fronteira constitui fator de proteção contra esta comorbidade.

No entanto, o agravamento das condições sociais e econômicas resulta em degradação significativa das condições de vida, aumentando a vulnerabilidade e consequentemente o risco de adoecimento por tuberculose e HIV 40, havendo a necessidade de que a ocorrência dessa comorbidade na região de fronteira internacional de Mato Grosso do Sul seja amplamente investigada entre indivíduos com tuberculose.

Ações que visem ao diagnóstico precoce de HIV e à prevenção do adoecimento por tuberculose em populações pobres, com acesso limitado a serviços de saúde, não serão bem-sucedidas caso não se aplique uma abordagem integrada que busque reduzir as desigualdades sociais subjacentes 41 .

A adesão ao tratamento da tuberculose é um processo multidimensional, determinado pela inter-relação de fatores pertinentes ao indivíduo, à doença, às condições socioeconômicas ao tratamento propriamente dito, ao sistema de saúde e aos profissionais 31 .

Embora as taxas de abandono de tratamento em Mato Grosso do Sul tenham superado a meta preconizada pela OMS (máximo de 5\%) 3, e mais pronunciadamente na região de fronteira (abandono 1,5 vez maior do que na região não fronteiriça), na população indígena verificou-se taxa de abandono próxima à máxima preconizada.

O elevado abandono $(13,1 \%)$ na população não indígena da fronteira pode advir de dificuldades na adesão ao tratamento, incluindo fatores como tabagismo, uso de drogas e bebidas alcoólicas, baixa escolaridade, efeitos adversos dos agentes quimioterápicos, coinfecção por HIV e abandono prévio, além de outras desvantagens socioeconômicas ${ }^{42}$. Na população indígena, a despeito da precariedade das condições socioeconômicas e outros agravantes, a cobertura oferecida pela Estratégia Saúde da Família (ESF) e 
pela ampliação do Tratamento Diretamente Observado (TDO) tem sido mostrado efetiva 35,36 o que possivelmente explica a associação entre ser indígena e não abandonar o tratamento de tuberculose pulmonar. Esse sucesso pode estar associado à adequada infraestrutura dos serviços ofertados pelo Distrito Sanitário Especial Indígena de Mato Grosso do Sul (DSEI-MS), à disponibilidade de recursos humanos (em termos de qualificação, número suficiente e baixa rotatividade) e ao envolvimento da comunidade nas ações de controle da doença 43 .

O sucesso no tratamento está diretamente relacionado com um melhor desempenho do Programa de Controle da Tuberculose, o que significa que pacientes em TDO que recebem acompanhamento adequado ao longo do curso terapêutico apresentam maiores índices de cura, menor abandono dos serviços de saúde e menor risco de evolução para complicações e óbitos $36,44,45$.

Isso foi confirmado em estudos sobre a população indígena do Amazonas 39, em que deficiências de serviços no acompanhamento sistemático dos casos elevaram em quase 12 vezes os índices de abandono do tratamento, em comparação com indivíduos que foram acompanhados com baciloscopias aos dois, quatro e seis meses de tratamento 25,32.

Nos municípios situados na linha de fronteira de Mato Grosso do Sul, o abandono do tratamento deve ser avaliado sob diferentes aspectos: endereços imprecisos ou situados fora da jurisdição brasileira, local de trabalho diferindo do local de moradia, residência temporária e dificuldades de acesso a serviços para acompanhamento do tratamento. Na tríplice fronteira Brasil/ Paraguai/Argentina, a organização da atenção básica que atende à população adstrita não facilitou o dignóstico precoce da tuberculose. Nessas circunstâncias, a propalada equidade torna-se de difícil dimensionamento, impossibilitando adscrever toda a clientela em um espaço delimitado e tornando parcial o alcance na atenção primária em saúde 17. A cobertura pela ESF mostrou-se insuficiente para assegurar a toda a população uma interação mais próxima e duradoura entre usuários e serviços, com vínculo e acolhimento. Aponta-se a necessidade de políticas específicas para as regiões de fronteira que garantam o direito universal à saúde, por meio de capacitação de recursos humanos e aporte financeiro para a organização dos serviços para o adequado enfrentamento da tuberculose nas Américas ${ }^{17}$.

\section{Considerações finais}

Os resultados obtidos devem ser considerados tendo-se em conta as limitações próprias da vigilância, como a subnotificação de casos e óbitos, e as inconsistências entre as bases de dados utilizadas, assim como o fato de terem sido analisados somente casos de tuberculose pulmonar. No entanto, os achados possibilitaram conhecer a situação epidemiológica da tuberculose pulmonar em Mato Grosso do Sul, permitindo identificar a área e a população em que o risco de adoecimento é maior, bem como avaliar de forma indireta a qualidade da assistência à população acometida por tuberculose pulmonar.

Conclui-se que em Mato Grosso do Sul a população que reside na região de fronteira encontra-se mais afetada por tuberculose pulmonar e com maior risco de abandoar o tratamento e morrer pela doença. Indivíduos indígenas apresentam maior risco de adoecer e morrer por tuberculose pulmonar, a despeito do menor abandono de tratamento, ao passo que a população não indígena apresenta maior coinfecção por HIV e maior grau de abandono do tratamento. Estar na região de fronteira é fator de proteção contra coinfecção por HIV. Não se constatou associação entre ser ou não indígena e coinfeção por HIV.

A tuberculose pulmonar é um problema de saúde pública em Mato Grosso do Sul, com maior magnitude nos municípios da fronteira internacional, o que salienta a necessidade de se empreenderem outras investigações que não só ampliem, mas aprofundem o conhecimento sobre fatores associados ao abandono do tratamento por indivíduos não indígenas, além de focalizarem outros preditores de morbimortalidade.

Para tanto, faz-se necessário ter em conta as peculiaridades locais e reorganizar as ações de vigilância em saúde considerando a sua complexidade, para efetivo gerenciamento da prevenção e do controle. É necessário também que as autoridades sanitárias elaborem e incorporem estratégias específicas e integradas que visem ao equacionamento desse importante e negligenciado problema de saúde pública. 


\section{Resumen}

Este estudio examinó la magnitud de la tuberculosis pulmonar del 2007 al 2010 en los municipios de Mato Grosso do Sul (Brasil) limítrofes con Paraguay y Bolivia. En la región fronteriza, las tasas de incidencia $(49,1 / 100.000$ habitantes), mortalidad $(4,0 / 100.000) y$ abandono del tratamiento (11,3\%) fueron 1,6, 1,8 y 1,5 veces más altas que en la región no fronteriza. En la región fronteriza, las tasas de incidencia $(253,4 / 100.000$ habitantes), mortalidad (11,6/100.000) y co-infección por el VIH $(1,9 / 100.000)$ entre residentes indígenas fueron 6,4, 3,2 y 1,9 veces más altas que en la población no indígena. Vivir en la región fronteriza resultó ser un factor protector contra la co-infección por el VIH. Se identificó asociación entre ser indígena y no abandonar el tratamiento. Los resultados mostraron que la población de estos municipios fronterizos está sujeta a un alto riesgo de enfermedad, muerte y abandono del tratamiento de la tuberculosis pulmonar, lo que requiere acciones de vigilancia de la salud específicas para este contexto.

Tuberculosis Pulmonar; Indios Sudamericanos; Áreas Fronterizas

\section{Colaboradores}

M. Marques contribuiu na concepção do projeto, levantamento, tabulação, interpretação dos dados, redação do artigo e aprovação da versão a ser publicada A. Ruffino-Netto participou da concepção do projeto, interpretação dos dados e avaliação crítica do artigo. A. M. C. Marques participou da interpretação dos dados, redação e revisão crítica do artigo. S. M. O. Andrade contribuiu na concepção do projeto, interpretação dos dados, redação do artigo e aprovação da versão a ser publicada. B. A. K. Silva e E. R. J. C. Pontes participaram da reorganização e análise estatística dos dados, bem como da discussão e redação do artigo.

\section{Agradecimentos}

À Ângela M. Marques pelo apoio nas estimativas populacionais indígenas. Aos colegas Maria do Socorro N Evangelista, Eunice A. T. Cunha e Rivaldo V. Cunha pela parceria na execução do projeto. À CNCPT/Ministério da Saúde pela aprovação do Projeto, parte deste estudo. À Divisão de Acompanhamento de Convênios/UFMS pelo gerenciamento do recurso. Ao Núcleo Estadual do Ministério da Saúde pela viabilização do recurso. Ao Fundo Nacional de Saúde pelo financiamento do Termo de Cooperação Técnica no 120/2010.

\section{Referências}

1. Barreira D, Grangeiro A. Avaliação das estratégias de controle da tuberculose no Brasil. Rev Saúde Pública 2007; 41 Suppl 1:4-8.

2. Conde MB, Melo FA, Marques AM, Cardoso NC, Pinheiro VG, Dalcin PT, et al. III Brazilian Thoracic Association Guidelines on tuberculosis. J Bras Pneumol 2009; 35:1018-48.

3. World Health Organization. Global tuberculosis report 2013. Geneva: World Health Organization; 2013.
4. Secretaria de Vigilância em Saúde, Ministério da Saúde. Especial tuberculose. Boletim Epidemiológico 2012; 43:1-12.

5. Ocampos AT. A saúde nas fronteiras: "uma proposta de bem-estar e desenvolvimento". In: Souza ML, Ferreira LAP, Rezende VM, Branco ML, organizadores. A saúde e inclusão social nas fronteiras. Florianópolis: Fundação Boiteux; 2008. p. 149-54. 
6. Gadelha CAG, Costa L. Integração de fronteiras: a saúde no contexto de uma política nacional de desenvolvimento. Cad Saúde Pública 2007; 23 Suppl 2:214-26.

7. Coordenação Geral de Doenças Endêmicas, Área Técnica de Pneumologia Sanitária, Departamento de Vigilância Epidemiológica, Secretaria de Vigilância em Saúde, Ministério da Saúde. Guia para cálculo de indicadores básicos e de avaliação da base de dados de tuberculose do Sistema de Informação de Agravos de Notificação - SINAN. Brasília: Ministério da Saúde; 2004.

8. Barcellos CC, Sabroza PC, Peiter P, Rojas LJ. Organização espacial, saúde e qualidade de vida: análise espacial e uso de indicadores na avaliação de situações de saúde. Inf Epidemiol SUS 2002; 11:12938.

9. Hino P, Villa TCS, Cunha TN, Santos CB. Distribuição espacial de doenças endêmicas no Município de Ribeirão Preto (SP). Ciênc Saúde Coletiva 2011; 16 Suppl 1:1289-94.

10. Oliveira GP, Torrens AW, Bartholomay P, Barreira D. Tuberculosis in Brazil: last ten years analysis 2001-2010. Braz J Infect Dis 2013; 2:218-33.

11. Corrêa LS. História e fronteira: o sul de Mato Grosso 1870-1920. Campo Grande: Editora da UCDB; 1999.

12. Serviço Brasileiro de Apoio às Micro e Pequenas Empresas, organizador. Mato Grosso do Sul sem fronteiras: características e interações territoriais: Brasil, Bolívia, Paraguai. Campo Grande: Editora Visão/SEBRAE; 2010.

13. Peiter P. Geografia da saúde na faixa de fronteira continental do Brasil na passagem do milênio [Tese de Doutorado]. Rio de Janeiro: Instituto de Geociências, Universidade Federal do Rio de Janeiro; 2005.

14. Organização Pan-Americana da Saúde; Organização Mundial da Saúde. Saúde nas Américas: panorama regional e perfis de países. Washington DC: Organização Pan-Americana da Saúde; 2012.

15. Martinez VN. Equidade em saúde: o caso da tuberculose na comunidade de bolivianos no Município de São Paulo [Dissertação de Mestrado]. São Paulo: Faculdade de Saúde Pública, Universidade de São Paulo; 2010.

16. Braga JU, Herrero MB, Cuellar CM. Transmissão da tuberculose na tríplice fronteira entre Brasil, Paraguai e Argentina. Cad Saúde Pública 2011; 27:1271-80.

17. Silva-Sobrinho RA, Andrade RLP, Ponce MAS, Wysocki AD, Brunello ME, Scatena LM, et al. Retardo no diagnóstico da tuberculose em município da tríplice fronteira Brasil, Paraguai e Argentina. Rev Panam Salud Pública 2012; 31:461-8.

18. Pinheiro RS, Oliveira GP, Oliveira EXG, Melo ECP, Coeli CM, Carvalho MS. Determinantes sociais e autorrelato de tuberculose nas regiões metropolitanas conforme a Pesquisa Nacional por Amostra de Domicílios, Brasil. Rev Panam Salud Pública 2013; 34:446-51.
19. Hino P. Padrões espaciais da tuberculose associados ao indicador adaptado de condição de vida no Município de Ribeirão Preto [Tese de Doutorado]. Ribeirão Preto: Escola de Enfermagem de Ribeirão Preto, Universidade de São Paulo; 2007.

20. Cunha EA, Ferrazoli L, Riley LW, Basta PC, Honer MR, Maia R, et al. Incidence and transmission patterns of tuberculosis among indigenous populations in Brazil. Mem Inst Oswaldo Cruz 2014; 109:108-13.

21. Sacchi FP, Croda MG, Estevan AO, Ko AI, Croda J. Sugar cane manufacturing is associated with tuberculosis in an indigenous population in Brazil. Trans R Soc Trop Med Hyg 2013; 107:152-7.

22. Basta PC, Coimbra Jr. CEA, Welch JR, Alves LCC, Santos RV, Camacho LAB. Tuberculosis among the Xavante Indians of the Brazilian Amazon: an epidemiological and ethnographic assessment. Ann Hum Biol 2010; 37:643-57.

23. Basta PC, Rios DPG, Alves LCC, Sant'Anna CC, Coimbra Jr. CEA. Estudo clínico-radiológico de crianças e adolescentes indígenas Suruí, Região Amazônica. Rev Soc Bras Med Trop 2010; 43: 719-22.

24. Croda MG, Trajber Z, Lima RC, Croda J. Tuberculosis control in a highly endemic indigenous community in Brazil. Trans R Soc Trop Med Hyg 2012; 106:223-9.

25. Belo EN, Orellana JDY, Levino A, Basta PC. Tuberculose nos municípios amazonenses da fronteira Brasil-Colômbia-Peru-Venezuela: situação epidemiológica e fatores associados ao abandono. Rev Panam Salud Pública 2013; 34:321-9.

26. Zevallos K, Vergara KC, Vergara A, Vidal C, Garcia $\mathrm{HH}$, Evans CA. Tuberculin skin-test reactions are unaffected by the severity of hyperendemic intestinal helminth infections and co-infections. Am J Trop Med Hyg 2010; 83:319-25.

27. Ladefoged K, Rendal T, Skifte T, Andersson M, Soborg B, Koch A. Risk factors for tuberculosis in Greenland: case-control study. Int J Tuberc Lung Dis 2011; 15:44-9.

28. Tollefson D, Bloss E, Fanning A, Redd JT, Barker K, McCray E. Burden of tuberculosis in indigenous peoples globally: a systematic review. Int J Tuberc Lung Dis 2013; 17:1139-50.

29. van der Werf MJ, Chechulin J, Yegorova OB, Marcinuk T, Stopolyanskiy A, Voloschuk V, et al. Health care seeking behaviour for tuberculosis symptoms in Kiev City, Ukraine. Int J Tuberc Lung Dis 2006; 10:390-5.

30. Basnet R, Hinderaker SG, Enarson D, Malla P, Mørkve O. Delay in the diagnosis of tuberculosis in Nepal. BMC Public Health 2009; 9:236.

31. World Health Organization. Global tuberculosis control 2011: WHO report 2011. Geneva: World Health Organization; 2011.

32. Rios DPG, Malacarne J, Alves LCC, Sant'Anna CC, Camacho LAB, Basta PC. Tuberculose em indígenas da Amazônia brasileira: estudo epidemiológico na região do Alto Rio Negro. Rev Panam Salud Pública 2013; 33:22-29. 
33. Ribas DL, Philippi ST. Aspectos alimentares e nutricionais de mães e crianças indígenas Terena, Mato Grosso do Sul. In: Coimbra Jr. CEA, Santos RV, Escobar AL, organizadores. Epidemiologia e saúde dos povos indígenas. Rio de Janeiro: Editora Fiocruz/ABRASCO; 2003. p. 73-88.

34. Morais MB, Alves GMS, Fagundes-Neto U. Estado nutricional de crianças índias Terenas: evolução do peso e estatura e prevalência atual de anemia. J Pediatr (Rio J.) 2005; 81:383-9.

35. Coordenação Regional do Mato Grosso do Sul, Fundação Nacional de Saúde. Relatório de atividades desenvolvidas na saúde indígena. Campo Grande: Fundação Nacional de Saúde; 2007.

36. Orellana JDY, Gonçalves MJF, Basta PC. Características sociodemográficas e indicadores operacionais de controle da tuberculose entre indígenas e não indígenas de Rondônia, Amazônia Ocidental, Brasil. Rev Bras Epidemiol 2012: 4:714-24.

37. Giovanella L, Guimarães L, Nogueira VMR, Lobato LVC, Damacena GN. Saúde nas fronteiras: acesso e demandas de estrangeiros e brasileiros não residentes ao SUS nas cidades de fronteira com países do MERCOSUL na perspectiva dos secretários municipais de saúde. Cad Saúde Pública 2007; 23 Suppl 2:S251-66.

38. Levino A, Carvalho EF. Análise comparativa dos sistemas de saúde da tríplice fronteira: Brasil/ Colômbia/Peru. Rev Panam Salud Pública 2011; 30:490-500.
39. Chirinos NEC, Meirelles, BHS. Fatores associados ao abandono do tratamento da tuberculose: uma revisão integrativa. Texto \& Contexto Enferm 2011; 20:399-406.

40. Girardi E. Epidemiology and control of tuberculosis in Italy. G Ital Med Lav Ergon 2010; 32:256-9.

41. Guimarães RM, Lobo AP, Siqueira EA, Borges TFF, Melo SCC. Tuberculose, HIV e pobreza: tendência temporal no Brasil, Américas e mundo. J Bras Pneumol 2012; 38:511-7.

42. Domingos MP, Caiaffa WT, Colosimo EA. Mortality, TB/HIV co-infection, and treatment dropout: predictors of tuberculosis prognosis in Recife, Pernambuco State, Brazil. Cad Saúde Pública 2008; 24:887-96.

43. Basta PC, Marques M, Oliveira RL, Cunha EAT, Resendes APC, Souza-Santos R. Desigualdades sociais e tuberculose: análise segundo raça/cor, Mato Grosso do Sul. Rev Saúde Pública 2013; 47: 854-64.

44. Pio A, Luelmo F, Kumaresan J, Spinaci S. National tuberculosis programme review: experience over the period 1990-95. Bull World Health Organ 1997; 75:569-81.

45. Raviglione MC, Pio A. Evolution of WHO policies for tuberculosis control, 1948-2001. Lancet 2002; 359:775-80.

Recebido em 09/Nov/2013

Versão final reapresentada em 17/Jun/2014

Aprovado em 10/Jul/2014 